\title{
Wireless point-of-care ultrasound: First experiences with a new generation handheld device
}

\author{
E.M. Jung ${ }^{\mathrm{a}, *}$, J. Dinkel $^{\mathrm{a}}$, N. Verloh ${ }^{\mathrm{a}}$, M. Brandenstein ${ }^{\mathrm{a}}$, C. Stroszczynski ${ }^{\mathrm{a}}$, \\ F. Jung ${ }^{\mathrm{b}}$ and J. Rennert ${ }^{\mathrm{a}}$ \\ anstitute of Diagnostic Radiology and Interdisciplinary Ultrasound Department, University Hospital, \\ Regensburg, Germany \\ ${ }^{\mathrm{b}}$ Institute of Biotechnology, Brandenburg University of Technology, Senftenberg, Germany
}

\begin{abstract}
.
AIM: To evaluate the diagnostic reliability of a new generation wireless point-of care ultrasound device for abdominal and thoracic findings.

MATERIAL AND METHODS: 40 patients (16 females, 24 males 19 - 80 years, on average 56.1 years) were scanned by an experienced examiner using the new wireless Vscan Air device for abdominal and thoracic findings. The probe frequencies were $2-5 \mathrm{MHz}$ (convex probe) and 3-12 MHz for the linear probe. As a reference standard, all patients were also examined using high-end ultrasound (LOGIQ E9/LOGIQ E10). Results were interpreted independently by two examiners in consensus, also with regard to the image quality $(0-4$, from not assessable $=0$, to excellent 4$)$.

RESULTS: In all 40 patients (100\%) examination with conventional high-end ultrasound and the Vscan Air ultrasound device was feasible. Sensitivity, specificity, positive and negative predictive value for the diagnosis of abdominal and thoracic findings were $63.3 \%, 100 \%, 100 \%$, and $40 \%$, respectively. Most main diagnostic findings were detected using the mobile device compared to the high-end ultrasound. Limitations were found regarding characterization and classification of hepatic and renal tumorous lesions.

Image quality revealed mostly minor diagnostic limitations for the mobile device, mean $2.9(\mathrm{SD} \pm 0.300)$ and was excellent or with only minor diagnostic limitations for conventional high-end ultrasound, mean 3.25 (SD \pm 0.438 ).

CONCLUSION: Due to its easy application and its high diagnostic reliability, point-of-care ultrasound systems of the latest generation represent a valuable imaging method for the primary assessment of abdominal and thoracic findings, especially in patients on intensive care units or in emergency situations.
\end{abstract}

Keywords: Point-of-care ultrasound, Vscan Air, high-end ultrasound

\section{Introduction}

Ultrasound is the most widely used imaging technology worldwide. The advantage lies in the good availability, real-time imaging with high detail resolution. The decisive factor, however, is the equipment and probe technology combined with the experience of the examiner. In recent years, three areas of ultrasound technology development have emerged in particular; the very comprehensive, innovative and technically complex high-end technology, the broadly deployed reliable technology of conventional devices and the small bedside or point-of-care ultrasound (POCUS), as an effective acute

\footnotetext{
*Corresponding author: Prof Dr. Med Ernst Michael Jung, Interdisciplinary ultrasound Department, University Hospital Regensburg, Franz-Josef Strauß Alle 11, 93042 Regensburg, Germany. E-mail: ernst-michael.jung@ukr.de. 


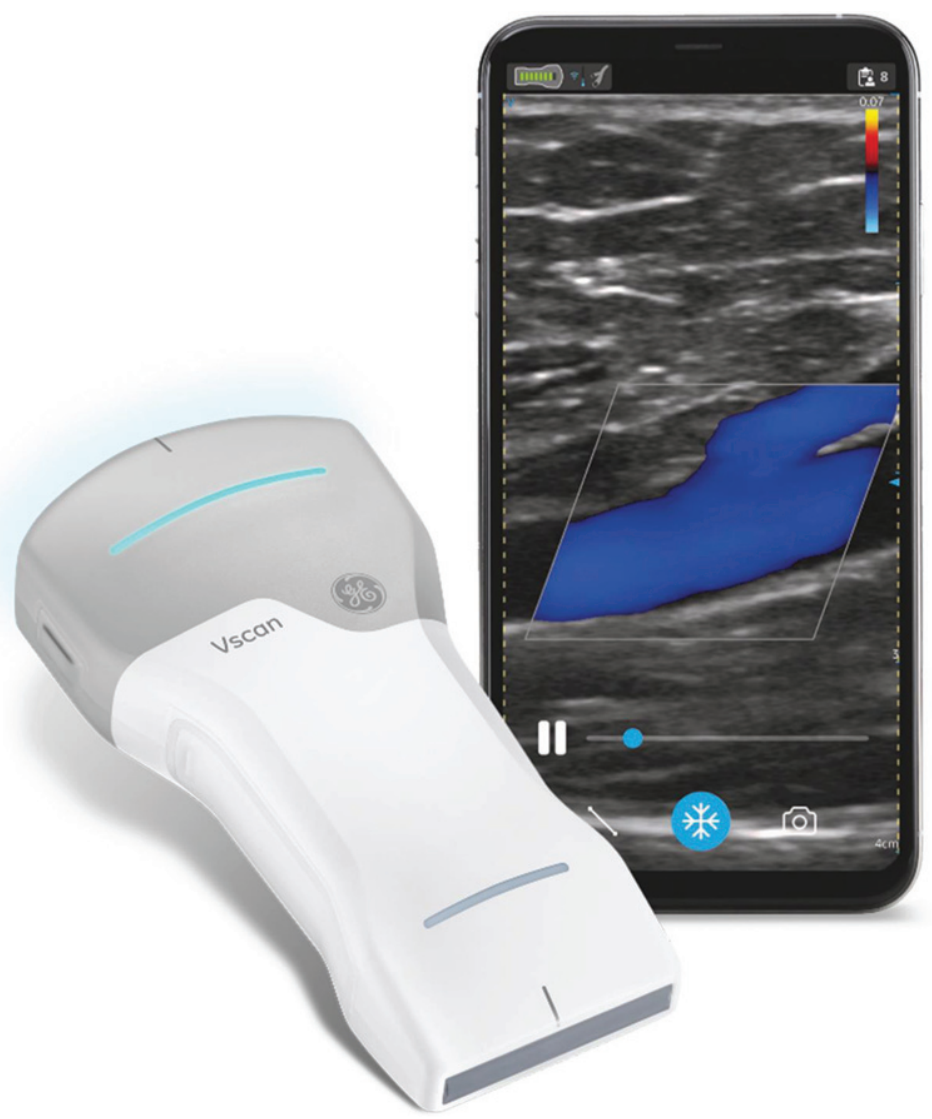

Fig. 1. Image of the wireless point-of care ultrasound device (Vscan Air, GE Healthcare, USA) with kind permission of Mr Benjamin Preissler (GE Healthcare).

diagnostic tool. The Vscan Air (GE Healthcare, USA, Fig. 1), a new 3rd generation point-of-care ultrasound device uses fundamental B-mode sonography and Color Coded Doppler Sonography (CCDS) in combination with a two-headed probe, convex for abdominal imaging and linear for vascular and soft tissue diagnostics to allow wide-ranging utilization.

In patients with blunt abdominal trauma, clinical assessment is difficult. Therefore, most algorithms rely heavily on fast imaging. Ultrasound examinations are carried out according to the Focused Assessment with Sonography for Trauma (FAST) guideline with standardized sections [1]. The aim is, to efficiently detect free abdominal fluid, pneumoperitoneum, pericardial effusion, pneumothorax or other life-threatening injuries. Regarding these questions, studies with mobile point of care ultrasound devices have been carried out successfully [2-7]. However, the flexibility of these previous devices was still limited, as the probe cable in particular restricts mobility and mobile data transmission is limited.

The point of care ultrasound devices are also considered for use in intensive care units, indications include cause of infection or reduced organ perfusion [8]. Especially in patients with Covid-19 infections, monitoring the extent of pulmonary consolidations and investigating possible abdominal inflammation with mobile ultrasound devices is much more efficient than the time-consuming and personnel-intensive transport to computed tomography (CT) [9].

In order to make point-off care ultrasound even more flexible, there are now devices with a two-headed probe that is wirelessly connected to an Android or iOS device via app. Scans 
Table 1

Basic demographic characteristics of the patients enrolled

\begin{tabular}{lcc}
\hline & $n$ & $\%$ \\
\hline $\begin{array}{l}\text { No. of patients } \\
\text { Gender }\end{array}$ & 40 & 100 \\
$\quad$ Male & 24 & 60 \\
$\quad$ Female & 16 & 40 \\
Median age and age range & $56.1(19-80)$ & 100 \\
Vscan Air exam & 40 & 100 \\
Conventional high-end ultrasound & 40 & 32.5 \\
ceCT & 13 & \\
\hline
\end{tabular}

are saved as single images or image sequences, also with the option of color-coded flow detection.

In order to evaluate the performance of this new mobile point-of-care technology, comparison with high-end ultrasound technology or contrast enhanced CT as gold standard is of interest. In addition, analysis of image quality with regard to challenging conditions e.g. meteorism or obesity is of importance [10].

In this pilot study, we evaluated the diagnostic yield and image quality of a new-generation wireless point-of-care ultrasound device compared to high-end ultrasound and contrast enhanced (ce)CT in patients with abdominal problems.

\section{Material and methods}

\subsection{Study design}

In April 2021, 40 consecutive patients (16 females, 24 males, age range 19-80 years, mean 56.1 years) registered for standard ultrasound examination with abdominal diseases were included in this retrospective study. All patients were examined using a new generation wireless point-of care ultrasound device (Vscan Air, GE Heathcare, USA). All patients were additionally examined using high-end ultrasound, in thirteen patients an additional ceCT was carried out. Basic demographic data of the patients enrolled in this study is shown in Table 1. Table 2 reveals the underlying diseases of the patients. The local ethics committee approved the study.

\subsection{Conventional high-end ultrasound}

Abdominal ultrasound examinations were carried out by an experienced examiner (more than 3000 examinations per year for more than 20 years) and digitally stored in our picture archiving and communication system (PACS) for independent evaluation. All patients were examined with conventional high-end sonography immediately after scanning with the mobile device using a multifrequency linear transducer (1-6 MHz, LOGIQ E9/LOGIQ E10, GE Healthcare).

First, the whole liver, spleen or kidney was examined using B-mode sonography in sweep technique. CCDS and Power Doppler (PD) ultrasound were used to evaluate native vascularization. Flow parameters were adjusted with modified pulse repetition frequency, color gain and wall filter and the best possible color imaging without blooming artifacts. Trauma patients were scanned according to the Focused Assessment with Sonography for Trauma 'FAST' guidelines [1] for the abdomen. Morison's, 
Table 2

Underlying disease of all patients

\begin{tabular}{lcc}
\hline Underlying disease & $n$ & $\%$ \\
\hline Melanoma & 3 & 7.5 \\
Myelodysplastic syndrome & 2 & 5 \\
Echinococcus multilocularis & 1 & 2.5 \\
Lymphoma/CML & 3 & 7.5 \\
Major trauma & 2 & 5 \\
Acute abdominal pain & 6 & 15 \\
Polycystic kidney disease & 2 & 5 \\
Previous liver/kidney transplant & 4 & 10 \\
Carcinoma & 4 & 10 \\
Hepatitis & 2 & 5 \\
Donor for Allo-Tx & 1 & 2.5 \\
Planned heart transplant & 1 & 2.5 \\
Covid-19 pneumonia & 2 & 5 \\
Fever of unknown origin & 2 & 5 \\
Liver cirrhosis & 2 & 5 \\
Osler disease & 1 & 2.5 \\
Waldenstroems macroglobulinemia & 1 & 2.5 \\
Renal cyst & 1 & 2.5 \\
\hline
\end{tabular}

Coller's and Douglas pouch as well as the pericardial sac were sonographically assessed and evaluated for free intra-abdominal fluid and organ lacerations.

For contrast-enhanced examinations with high-end sonography or computed tomography, contraindications were taken into account and written consent was obtained. Contrast enhanced ultrasound (CEUS) was performed after bolus injections of 1-2.4 $\mathrm{ml}$ of sulphur hexaflouride microbubbles (SonoVue ${ }^{\circledR}$, BRACCO, Italy) with a low mechanical index $(\mathrm{MI}<0.16)$ applying CEUS with amplitude modulation and pulse inversion harmonic imaging (PIHI) technique. The contrast harmonic imaging technique $(\mathrm{CHI})$ uses a contrast-specific detection mode for real-time evaluation of the contrast-agent enhancement.

Pulmonary inflammatory changes, especially in patients with COVID-19 infection, were assessed for various pathologies by examining the pleural line and subpleural regions and whether A or B lines were present. The findings of the independently assessed CT served as a reference.

\subsection{Vscan air point-of-care ultrasound (POCUS)}

All 40 patients were examined using a last generation wireless handheld ultrasound device. Vscan Air is a battery operated general-purpose diagnostic ultrasound imaging system. It enables ultrasound imaging, visualization and measurement of anatomical structures and fluids. Black-and-white mode is offered for displaying anatomy in real-time color-coded overlay for real-time blood flow imaging. Vscan Air consists of a two-headed probe that integrates both convex (2-5 MHz) and linear array transducers (3-12 MHz) and an app that can be installed on Android ${ }^{\mathrm{TM}}$ or $\mathrm{iOS} \circledast$ (iOS 13 or higher) mobile devices. Pocket-sized portability and simplified user interface allow integration into training and examinations in professional healthcare facilities. The device has a total scan time of 50 minutes with fully charged battery (with $80 \%$ black and white, $20 \%$ color imaging). The battery can be recharged from $10 \%$ to $90 \%$ in $75 \mathrm{~min}$. The dimensions are $131 \times 64 \times 31 \mathrm{~mm}$, the weight $205+/-3$ grams. 
The information can be used for basic/focused assessments and in addition to other medical data for clinical diagnostic purposes in routine, periodic follow-up, and triage assessments for adult, pediatric, and neonatal patients. Vscan Air customers have access to the Vscan web portal, including online access to product and product usage information for selected clinical scenarios. The image and patient data are stored in encrypted form on the mobile device and can be transmitted wirelessly via a DICOM connection.

The Vscan Air offers ultrasound imaging with a minimized number of buttons and an intuitive, thumb-operated touchscreen user interface. The Vscan Air app supports both portrait and landscape mode to optimize image size and ergonomics for various application scenarios. The internal memory requirement of the mobile device on which the Vscan Air is operated with the Vscan Air app should be at least $8 \mathrm{~GB}$. The data connection is made via Bluetooth BLE 4.0.

For abdominal examinations, parenchymal organs were scanned according to the clinical problem. Thus, organ images were documented biplanar using fundamental B-mode. For evaluation of the native vascularization, CCDS was used. These images were evaluated by two experienced examiners in consensus and compared to previous ultrasound or contrast enhanced computed tomography studies (CT).

\subsection{Computed tomography}

Thirteen patients received contrast-enhanced CT (ceCT) of the abdomen and pelvis in in portalvenous phase $(70-90 \mathrm{sec})$ with bolus injection of iodic contrast-agent $\left(80-130 \mathrm{ml}\right.$ Accupaque $^{\mathrm{TM}}$, GE) (SOMATOM Definition Flash Siemens Healthcare, Erlangen Germany; collimation 5 mm with coronary and axial reconstructions). Written informed consent was obtained by all patients and contraindications were taken into account. The ceCT scans were evaluated by two radiologists with an expertise in abdominal imaging of more than 15 and 20 years.

\subsection{Image quality}

To achieve the best assessment, an artifact free display of the pathology without overlapping of surrounding tissue was necessary. Image quality of the Vscan Air ultrasound device and the conventional high-end ultrasound was evaluated based upon a five points scale: 0: assessment not possible, e.g. due to technical problems, 1: non-diagnostic, 2: major diagnostic limitations, 3: minor diagnostic limitations, 4: excellent.

\subsection{Statistical analysis}

Data were acquired using Excel tables (Excel 2016, Microsoft, USA). Statistical analysis was performed using PASW 18 (PASW V.18, IBM SPSS Inc., Armonk, NY, USA). Agreement between the findings of point-of care ultrasound versus conventional ultrasound was assessed from $2 \times 2$ tables and Chi Square test.

\section{Results}

In all 40 patients, conventional high-end ultrasound and point- of care ultrasound was carried out successfully. The mean examination time for the mobile device was about 90 seconds with a range from 1-4 minutes. 
Table 3

Sensitivity, specificity, positive and negative predictive value for the diagnosis of abdominal and thoracic findings using Vscan Air and high-end ultrasound as standard of reference

\begin{tabular}{lc}
\hline & $\%$ \\
\hline Sensitivity & 63.3 \\
Specificity & 100 \\
Positive predictive value (PPV) & 100 \\
Negative predictive value (NPV) & 40 \\
Hit rate & 85.1 \\
\hline
\end{tabular}

The main findings in abdominal ultrasound scanning $(n=38)$ were splenomegaly $(n=9)$, splenic tumor ( $n=1$, Fig. 2), benign/malignant liver tumor ( $n=5$ Fig. 3$)$, hepatic abscess $(n=1$, Fig. 4$)$, free abdominal fluid ( $n=3)$, hematoma ( $n=2$, Fig. 5), intra-/extrahepatic cholestasis $(n=2)$, cholecystolithiasis $(n=2)$, hepatic steatosis $(n=3)$ renal cyst $(n=5)$, renal tumor $(n=2)$, inguinal hernia $(n=1)$, renal laceration $(n=1)$, diverticulitis $(n=2)$, thickening of the gastric wall $(n=1)$ and inconspicious abdominal exam $(n=4)$. Multiple findings occurred in several patients. In patients who underwent thoracic ultrasound $(n=2)$, main diagnostic findings were pleural effusion $(n=2)$ and pulmonal inflammatory changes $(n=2$, Fig. 6$)$. These findings were confirmed by ceCT if available.

The main abdominal findings, detected by conventional high-end ultrasound were confirmed using the mobile device in 34 out of 38 patients (89\%). In four patients $(10.5 \%)$, the standard of reference revealed no conspicuous abdominal findings, which was verified with the Vscan Air. In six patients (15\%), point-of care ultrasound only showed inexplicit hepatic and renal tumorous lesions, which remained inconclusive. Here, conventional high-end ultrasound with CEUS was able to classify these lesions as focal nodular hyperplasia, hemangioma, metastases, complex renal cyst and renal angiomyolipoma.

Conventional high-end ultrasound and the point-of care mobile device concordantly detected the main thoracic findings.

Overall, sensitivity, specificity, positive and negative predictive value of Vscan Air for the diagnosis of abdominal and thoracic findings were $63.3 \%, 100 \%, 100 \%$, and 40\%, respectively (Table 3).

In all 40 patients (100\%) examination with conventional high-end ultrasound and the Vscan Air ultrasound device was feasible. For the mobile device, image quality showed only minor diagnostic limitations in most patients $(n=36)$ and major diagnostic limitations in only 4 patients, mean 2.9 (SD \pm 0.300 , variability 0.092). Conventional high-end ultrasound revealed excellent or only minor diagnostic limitations, mean 3.25 ( $\mathrm{SD} \pm 0.438$, variability 0.1923). The differences were not statistically significant.

\section{Discussion}

In this pilot study, the significance of a new point-of-care ultrasound technique, wireless with smartphone connection, was underlined as a decisive step for even more mobility in imaging. Even with a limited number of examinations, the wide field of application of this new technology is indicated. On the one hand, the availability for focused assessment with sonography for trauma FAST examinations could be extended. In addition, evaluation for free abdominal fluid or pleural effusion on intensive care units or as a bedside examination is possible.

The new mobile technology quickly provides clues for potential abdominal inflammations. Furthermore, pneumonic consolidations in COVID-19 infections can be visualized. The easy handling 


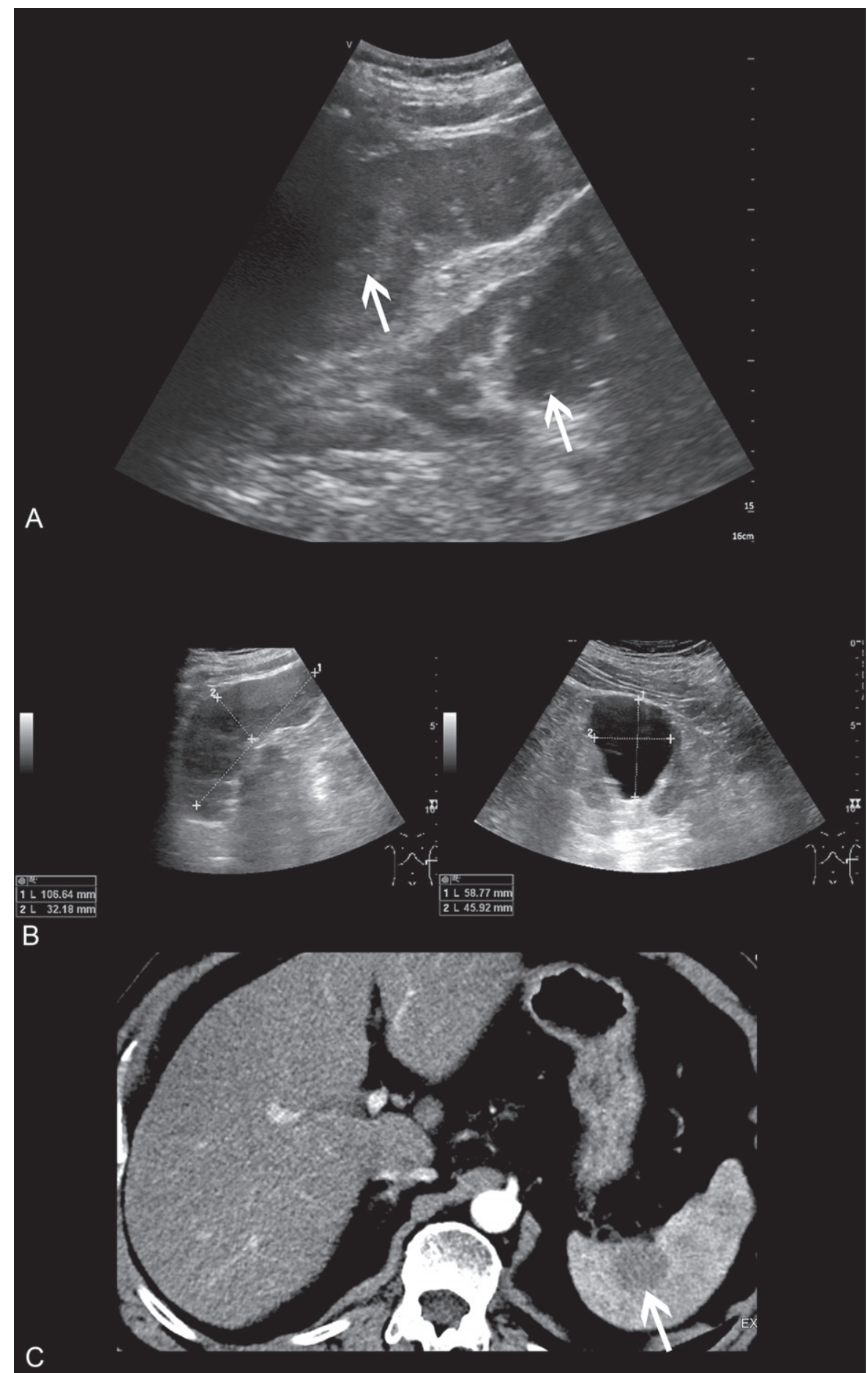

Fig. 2. 72 year old patient, diagnosed with T4 tonsillar carcinoma, who underwent CT for evaluation of metastatic lesions. A: Vscan image of the upper abdomen showed hypoechogenic splenic and renal lesions, marked with arrow. Image quality was rated as 2 due to blurring and low resolution. B: High-end ultrasound image confirmed the hypoechogenic renal and splenic lesions. Images are displayed with a much better resolution and quality (rated as 3). C: Corresponding CT scan of the upper abdomen that showed multiple obscure splenic hypodensities with diminished contrast enhancement. Pathologies are marked with an arrow. 


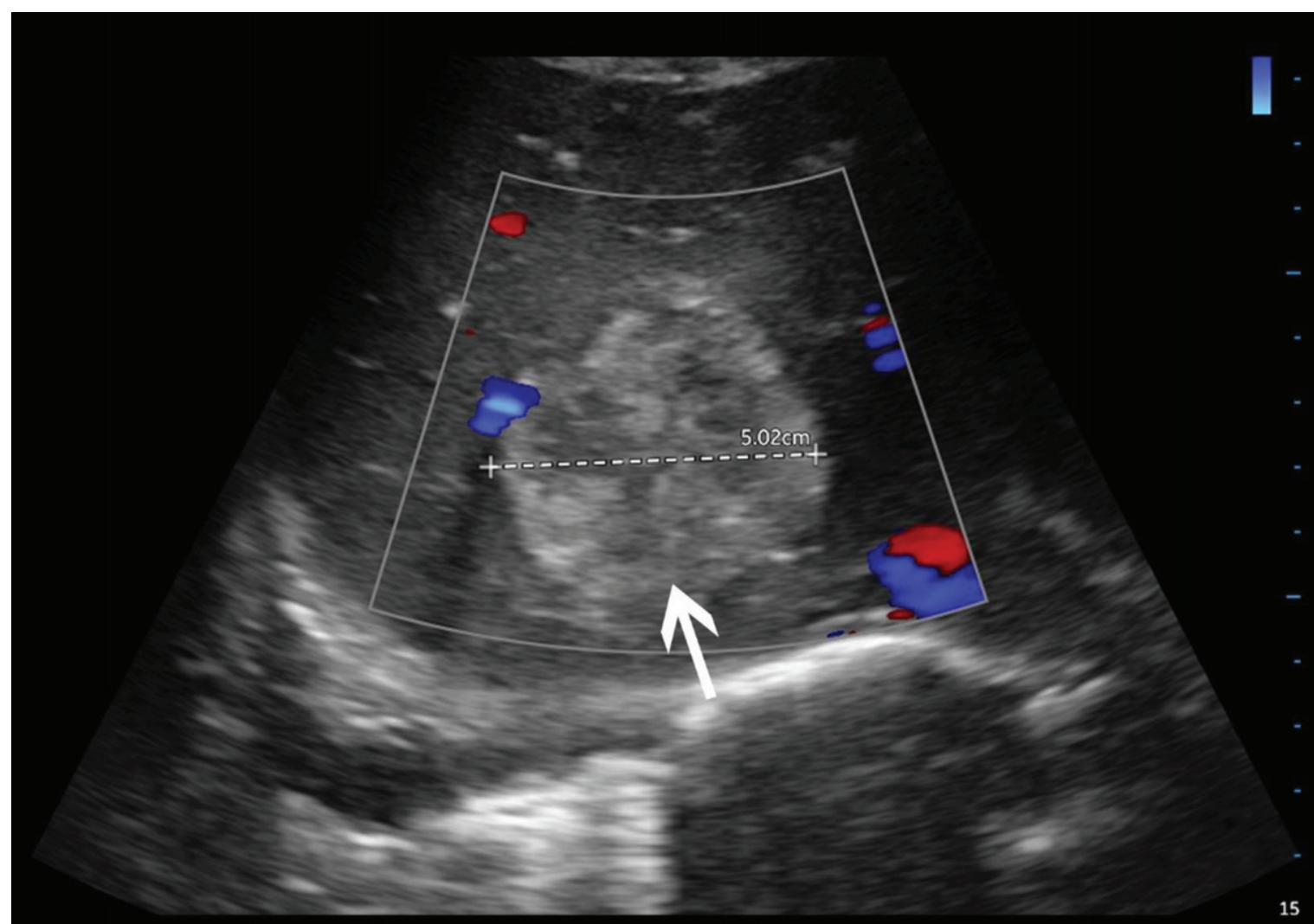

A
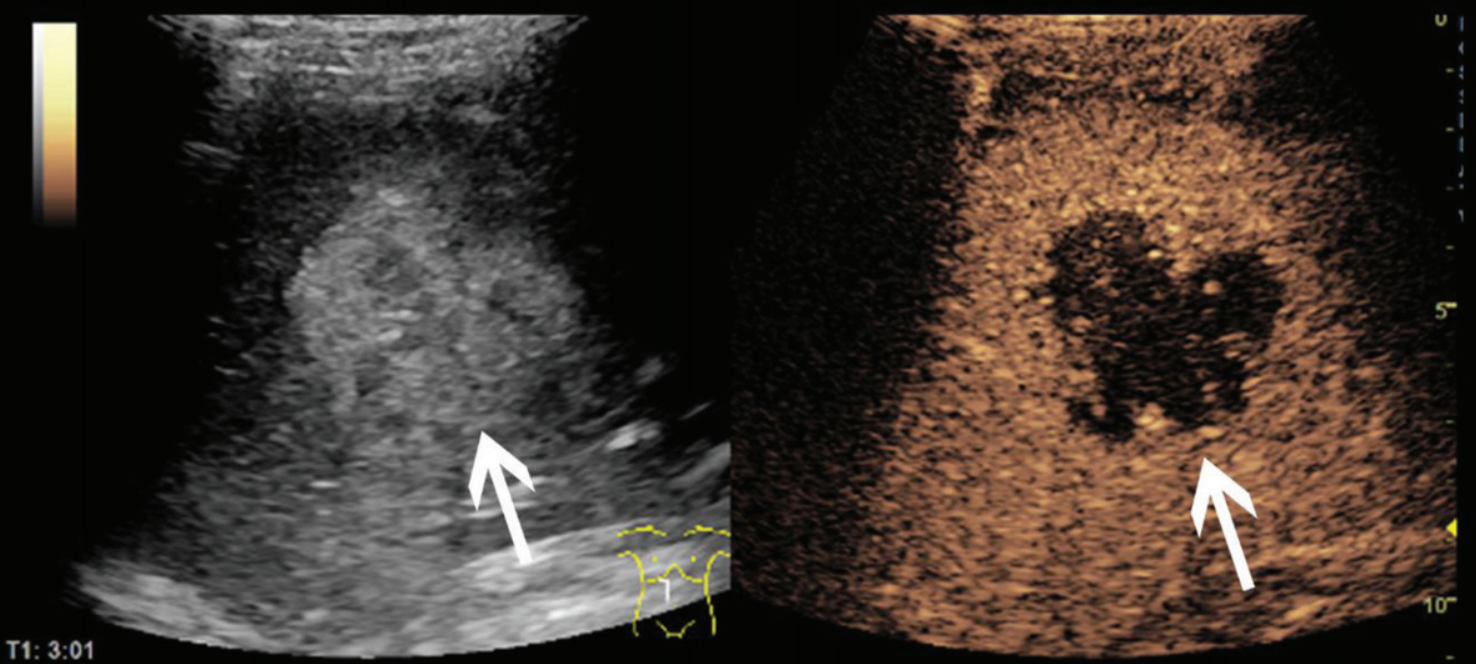

\section{B}

Fig. 3. 69 year old patient with newly diagnosed retroauricular malignant melanoma. A: Vscan ultrasound showed a 5 $\mathrm{cm}$ hyperechogenic hepatic lesion with no relevant perfusion on CCDS. Image quality was rated as 3 altogether. B: Highresolution ultrasound confirmed this lesion. CEUS revealed a typical nodular centripetal enhancement, consistent with a partially thrombosed hemangioma. Image quality was rated as excellent (4). Pathologies are marked with an arrow. 


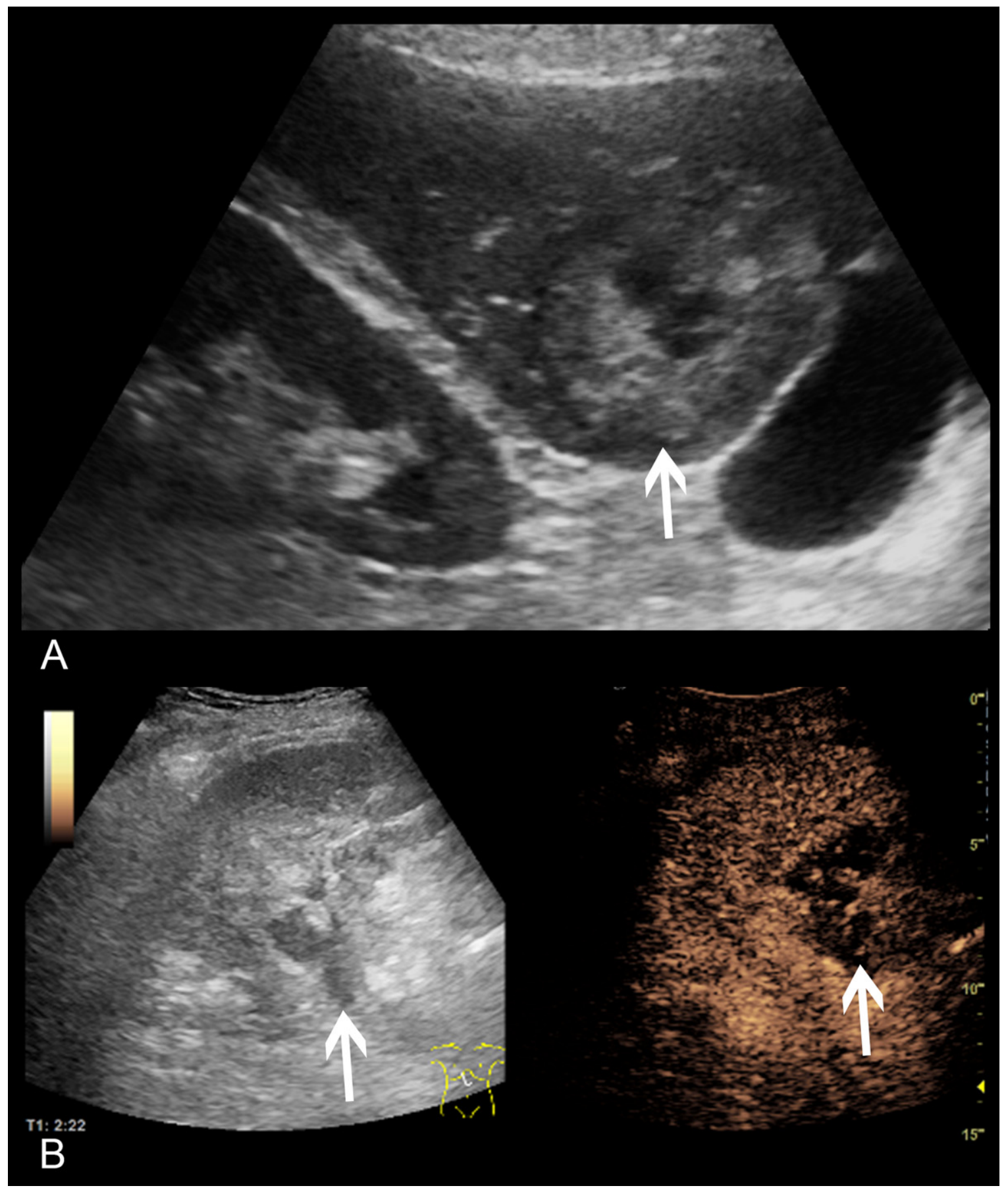

Fig. 4. 61 year old patient with previous liver transplantation, admitted to our hospital with fever of unknown origin. A: Vscan image showed a large partially hyperechogenic mass with central hypoechogenicity and adjacent free abdominal fluid. Image quality showed only minor diagnostics limitations (3). B: High-end ultrasound with CEUS revealed several hypoechogenic lesions with profound enhancement in between. In correlation with an MRI scan, multiple cholangitic abscesses were confirmed. Image quality was found to be excellent (4). Pathologies are marked with an arrow. 


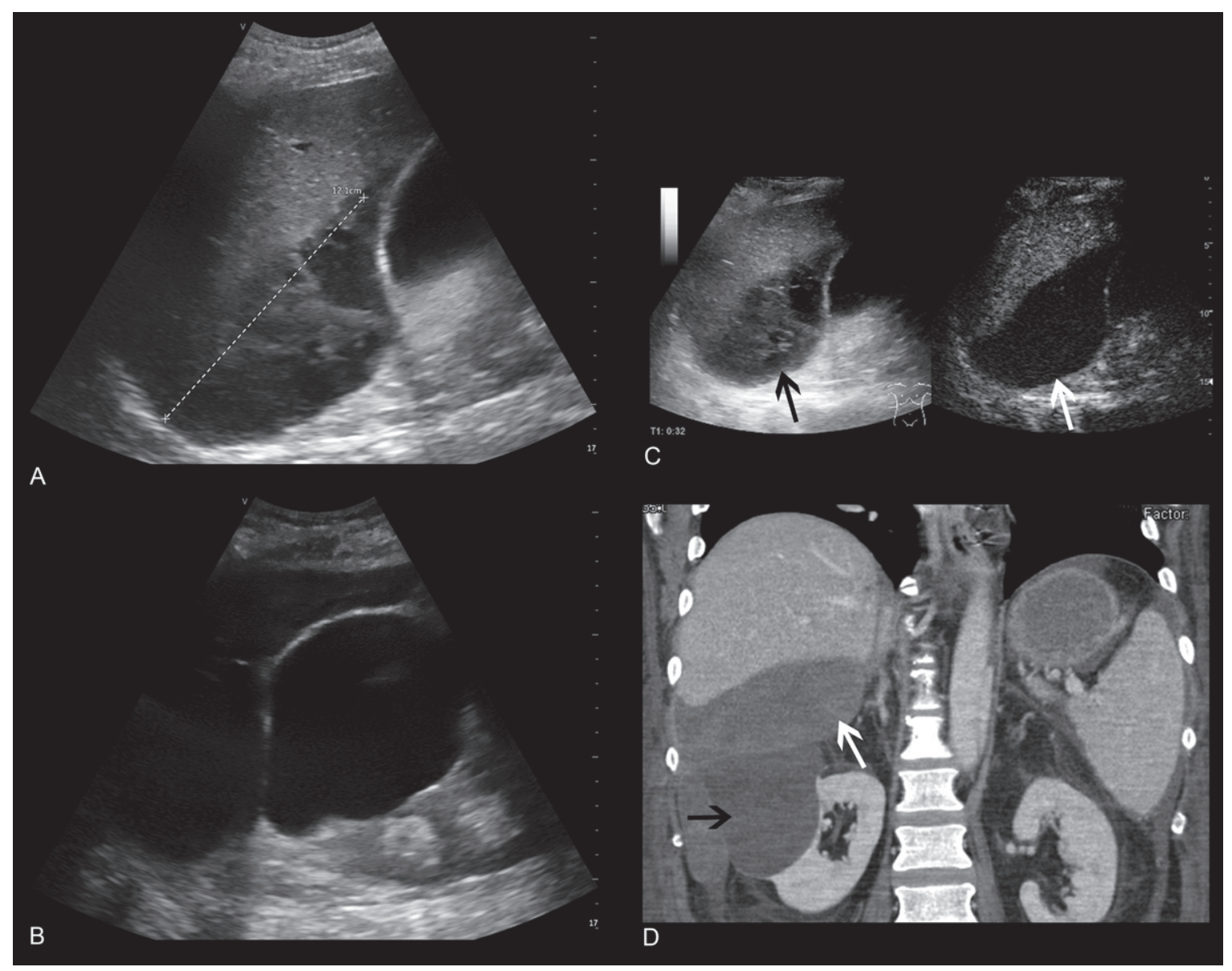

Fig. 5. 49 year old patient following liver transplantation with acute decrease in hemoglobin. A/B: Vscan images display an extensive subhepatic hematoma and a large renal cyst. Image quality showed only minor diagnostic limitations (3) C: High-end ultrasound image conformed the subhepatic hematoma as well as the adjacent renal cyst, clearly separated. Image quality of the exam was found to be excellent. D: On a ceCT scan for elimination of an acute bleeding, both pathologies were clearly visible. Pathologies are marked with an arrow.

and unpretentious implementation of hygiene protocols for the mobile wireless probe is particularly advantageous.

Regarding the image quality, the patient's constitution and compliance are of course decisive. While examinations in obese patients were susceptible to disruptions of the data transmission with previous handheld devices, the new wireless Vscan technology seems to be more stable, under the presumption of an adequate WI-FI connection. However, results of previous studies [11], that point-of care ultrasound allows rapid emergency diagnosis of free abdominal fluid, pneumoperitoneum and pericardial or pleural effusion can be confirmed.

The diagnostic reliability of ultrasound is strongly dependent upon the experience of the examiner, even more so in mobile devices. One limitation of the mobile device is a limited continuous scanning time of approx. 50 minutes with one battery charge. However, the examination time per patient was significantly reduced compared to earlier versions of the battery-operated handheld device. In order to guarantee the appropriate performance of the device, high performance smartphones based on the latest android or iOS system software are to be preferred. Patient registration, program structure for B-mode and CCDS, image storage, as well as image analysis are adjusted to the touch function of 


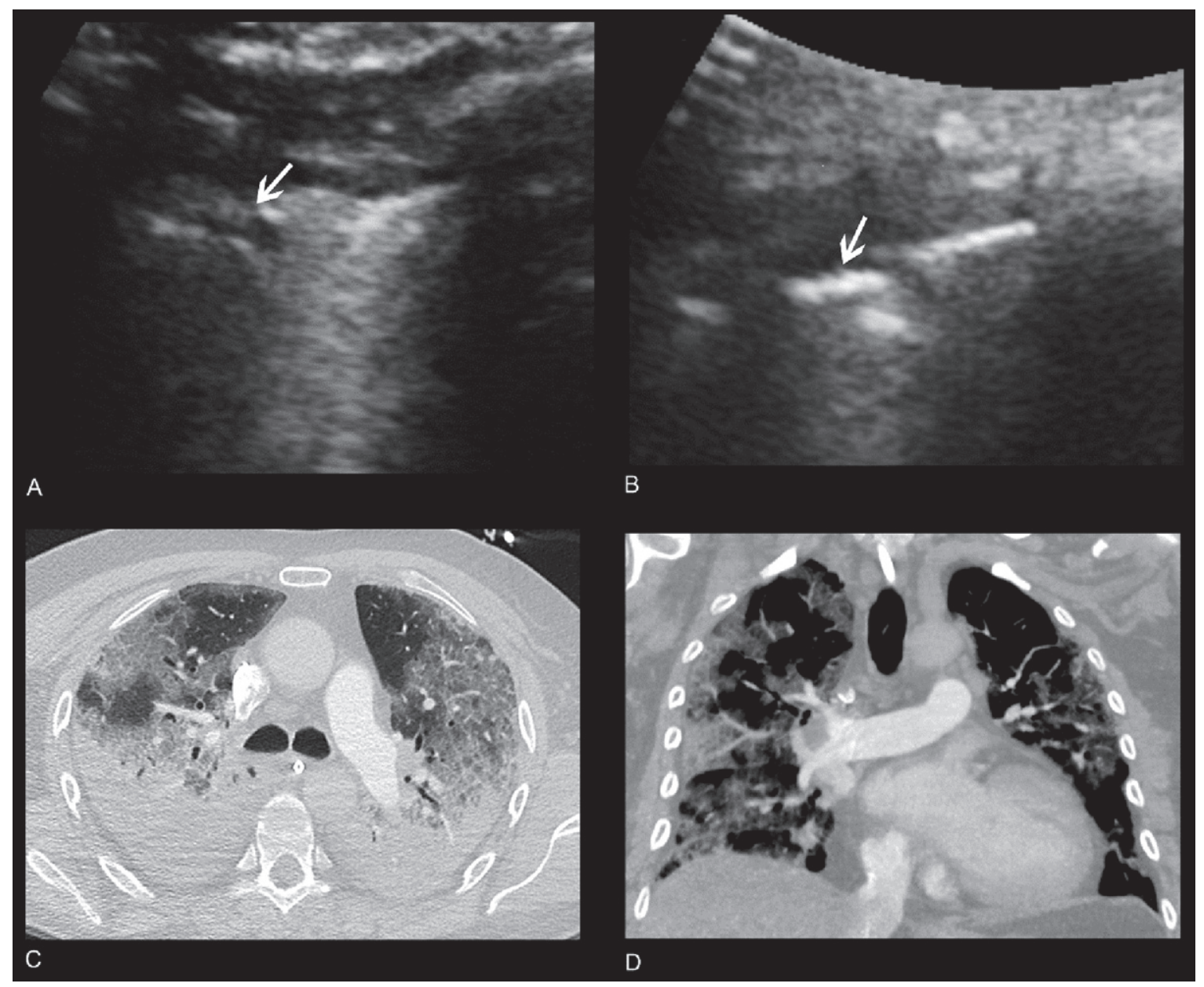

Fig. 6. 65 year old male patient with severe COVID-19 pneumonia. A/B: Vscan images using a transabdominal probe display consolidations with A- and B-lines. Image quality showed minor diagnostic limitations (2-3). C/D: CT images displaying small bilateral pleural effusions and diffuse, predominantly peripheral ground glass opacities, and peripheral consolidations consistent with COVID-19 pneumonia.

regular ultrasound devices. This leads to a faster and easily practicable workflow.

The image quality of the mobile device appears to be sufficient for fast abdominal ultrasound diagnostics. However, compared to high-end devices, the depth assessment and detailed analysis are inferior. One main limitation is that while Vscan technology can assess color flow, Doppler spectral analysis and continuous adaptation to various velocity ranges is not available. Only two velocity levels are provided for the mobile device, high level mean $30 \mathrm{~cm} / \mathrm{s}$ and low level mean $12 \mathrm{~cm} / \mathrm{s}$. This allows a rough evaluation of the arterial and venous perfusion, but no differentiated segmental assessment. The missing Doppler analysis might be disadvantageous, especially following organ transplantation. Nevertheless, definite identification of inflammation and reduced blood flow, but also of tumorous lesions detected by B-mode sonography is feasible using the new wireless VScan technology. Thus, an increasing utilization on intensive care units, in emergency imaging or in patients with infections seems reasonable.

However, since no Doppler spectral analysis, no elastography and no contrast enhanced ultrasound technology is integrated in the present mobile ultrasound devices, high-end ultrasound remains the imaging technique that allows an even more detailed analysis of abdominal and pulmonary pathologies 
including the evaluation of microcirculation, as current studies show [12-16]. Nevertheless, under the premise of proper training, POCUS shows advantages in fast and critical situations or in intensive care units.

\section{Conflicts of interest}

The authors have no potential conflicts of interest to report.

\section{References}

[1] Scalea TM, Rodriguez A, Chiu WC, Brenneman FD, Fallon WF JR, Kato K, et al. Focused Assessment with Sonography for Trauma (FAST): results from an international consensus conference. J Trauma. 1999;46(3):466-72. doi:10.1097/00005373-199903000-00022.

[2] Schleder S, Dendl L-M, Ernstberger A, Nerlich M, Hoffstetter P, Jung E-M, et al. Diagnostic value of a hand-carried ultrasound device for free intra-abdominal fluid and organ lacerations in major trauma patients. Emerg Med J. 2013;30(3):e20. doi:10.1136/emermed-2012-201258.

[3] Kirkpatrick AW, Breeck K, Wong J, Hamilton DR, McBeth PB, Sawadsky B, et al. The potential of handheld trauma sonography in the air medical transport of the trauma victim. Air Med J. 2005;24(1):34-9. doi:10. 1016/j.amj.2004.10.012.

[4] Kirkpatrick AW, Sirois M, Laupland KB, Goldstein L, Brown DR, Simons RK, et al. Prospective evaluation of hand-held focused abdominal sonography for trauma (FAST) in blunt abdominal trauma. Can J Surg. 2005;48(6):453-60.

[5] Piccoli M, Trambaiolo P, Salustri A, Cerquetani E, Posteraro A, Pastena G, et al. Bedside diagnosis and follow-up of patients with pleural effusion by a hand-carried ultrasound device early after cardiac surgery. Chest. 2005;128(5):341320. doi:10.1378/chest.128.5.3413.

[6] Schleder S, Jung EM, Heiss P, Stroszczynski C, Schreyer AG. Hand-carried and high-end ultrasound systems are equally inferior to abdominal radiography and multidetector computed tomography in the diagnosis of pneumoperitoneum. Rofo. 2014;186(3):219-24. doi:10.1055/s-0033-1356222.

[7] Schleder S, Dornia C, Poschenrieder F, Dendl L, Cojocaru L, Bein T, et al. Bedside diagnosis of pleural effusion with a latest generation hand-carried ultrasound device in intensive care patients. Acta Radiol. 2012;53(5):556-60. doi:10.1258/ar.2012.110676.

[8] Baribeau Y, Sharkey A, Chaudhary O, Krumm S, Fatima H, Mahmood F, Matyal R. Handheld Point-of-Care Ultrasound Probes: The New Generation of POCUS. J Cardiothorac Vasc Anesth. 2020 Nov;34(11):3139-45. doi: 10.1053/j.jvca.2020.07.004.

[9] Bianchi S, Savinelli C, Paolucci E, Pelagatti L, Sibona E, Fersini N, et al. Point-of-care ultrasound (PoCUS) in the early diagnosis of novel coronavirus 2019 disease (COVID-19) in a first-level emergency department during a SARS-CoV-2 outbreak in Italy: a real-life analysis. Intern Emerg Med. 2021. doi:10.1007/s11739-021-02643-w.

[10] Lee L, DeCara JM. Point-of-Care Ultrasound. Curr Cardiol Rep. 2020;22(11):149. doi:10.1007/s11886-020-01394-y.

[11] Guevarra K, Greenstein Y. Ultrasonography in the Critical Care Unit. Curr Cardiol Rep. 2020;22(11):145. doi:10. 1007/s11886-020-01393-Z.

[12] Han H, Ji Z, Ding H, Zhang W, Zhang R, Wang W. Assessment of blood flow in the hepatic tumors using non-contrast micro flow imaging: Initial experience. Clin Hemorheol Microcirc. 2019;73(2):307-16. doi:10.3233/CH-180532.

[13] Wiesinger I, Jung F, Jung EM. Contrast-enhanced ultrasound (CEUS) and perfusion imaging using VueBox®. Clin Hemorheol Microcirc. 2021. doi:10.3233/CH-201040.

[14] Schwarze V, Marschner C, Völckers W, Figueiredo GN de, Rübenthaler J, Clevert D-A. The diagnostic performance of contrast-enhanced ultrasound (CEUS) for evaluating hepatocellular carcinoma (HCC) juxtaposed to MRI findings; a retrospective single-center analysis of 292 patients. Clin Hemorheol Microcirc. 2020;76(2):155-60. doi:10.3233/CH209213.

[15] Zhang H-J, Zheng B-W, Gu S-J, Wu T, Wu L-L, Lian Y-F, et al. Doppler ultrasonography and contrast-enhanced ultrasonography to evaluate liver allograft discard: A pilot prospective study. Clin Hemorheol Microcirc. 2021;77(1):107-14. doi:10.3233/CH-200950.

[16] Shao S, Yao M, Li X, Li C, Chen J, Li G, et al. Conventional and contrast-enhanced ultrasound features in sclerosing adenosis and correlation with pathology. Clin Hemorheol Microcirc. 2021;77(2):173-81. doi:10.3233/CH-200943. 